\title{
Fabrication of autocloned photonic crystals by using high-density-plasma chemical vapor deposition
}

\author{
H. L. Chen ${ }^{\text {a) }}$ \\ Department of Materials Science and Engineering, National Taiwan University, Taipei, Taiwan, \\ Republic of China \\ H. F. Lee \\ National Tsing Hua University, Hsinchu, Taiwan, Republic of China
}

W. C. Chao, C. I. Hsieh, and F. H. Ko

National Nano Device Lab., 1001-1 Ta Hsueh Road Hsinchu, Taiwan, Republic of China

T. C. Chu

National Tsing Hua University, Hsinchu, Taiwan, Republic of China

(Received 25 June 2004; accepted 4 October 2004; published 17 December 2004)

\begin{abstract}
The high-density-plasma chemical vapor deposition (HDP-CVD) method was demonstrated as an alternative to radio-frequency (rf) bias sputtering method for fabrication of "autocloned" photonic crystals. We successfully preserved periodic surface corrugation after deposition of multilayer stacks under appropriate chemical vapor deposition conditions. Freedom of the shaping process was increased by simply raising the bias power of the autocloning process, and thus created autocloned structures having a strong modulation of the effective refractive index in the lateral direction. The method allows photonic bands of autocloned photonic crystals to be designed with greater controllability and a simpler fabrication process. Furthermore, the HDP-CVD method has better step-coverage than the sputtering method and can be used to fabricate autocloning structures with small feature-sizes. (ㅇ 2004 American Vacuum Society. [DOI: 10.1116/1.1824059]
\end{abstract}

\section{INTRODUCTION}

Photonic crystals $(\mathrm{PhCs})$, which are the artificial multidimensional periodic structures composed of two kinds of materials, are attractive materials for ultra-compact light wave circuits. ${ }^{1-5}$ Various photonic crystals devices, based on their three main characteristics: photonic band gap, strong optical anisotropy and high dispersion, have been reported such as waveguides, polarization splitters, and superprisms. ${ }^{6-12}$ In recent years, several methods for fabricating three-dimensional (3D) photonic crystals with full 3D band gaps have been reported. One of the methods used to fabricate $3 \mathrm{D}$ periodic structures is alternating-layer deposition on a two dimensional (2D)-patterned substrate. ${ }^{1}$ However the conventional alternating-layer deposition of plasma enhanced chemical vapor deposition (PECVD) leads to a rapid flattening of the corrugated structure and thus does not yield a 3D periodic structure. ${ }^{13}$ Kawakami developed a simple fabrication process called "autocloning technology," which is based on the deposition of multilayer wavy films onto a substrate with periodically arrayed holes or grooves by a combination of electron-beam lithography, dry etching and radio-frequency (rf) bias sputtering. ${ }^{3}$ The autocloning method successfully solved the flattening problem, and made it possible to fabricate a large-scale 3D periodic structure. .,10,12 $^{9}$

In this article, a high-density-plasma chemical vapor deposition (HDP-CVD) method with inductively coupled plasma (ICP)-sourced was used as an alternative to the rf-

${ }^{a)}$ Electronic mail: hsuenlichen@ccms.ntu.edu.tw bias sputtering method for fabrication of autocloned photonic crystals. $\mathrm{Si} / \mathrm{SiO}_{2}$ films were used in alternating multilayer structures. We successfully preserved the periodic surface corrugation after deposition of multilayer structures under appropriate chemical vapor deposition conditions. We also investigated several process factors involved in the selfshaping and autocloning mechanisms. Moreover we increased the freedom of the shaping process by adjusting the substrate power during the autocloning process without any other additional equipment such as a reactive ion etcher (RIE). This method can create autocloned structures having a strong modulation of the effective refractive index in the lateral direction by a simple fabrication process.

\section{EXPERIMENT SETUP}

The HDP-CVD system (Duratek, Mutiplex Cluster) with ICP-source has a chamber surrounded by rf coils and a rfbias provided for substrates. The former enables a highdensity plasma with a large ion content and the latter can control the energy of ion-bombardment through a dc bias arises. Although the deposition mechanism between HDPCVD and sputtering is quite different, both of them have angle-selective sputter etching effect due to rare ions accelerated by rf-bias along with deposition process. On the other hand, the ICP can retain high-density plasma at very low pressure $\left(10^{-5}-10^{-6}\right.$ Torr) and thus increase the mean-freepath of arriving ions. This can lead to better step coverage and conformality and can be used on smaller patterned autocloned phtonic crystals. In addition, HDP-CVD has a multi- 
TABLE I. Deposition conditions of autocloned photonic crystals composed of $a$-Si and $\mathrm{SiO}_{2}$ films by the HDP-CVD.

\begin{tabular}{ccc}
\hline \hline & Si deposition & $\mathrm{SiO}_{2}$ deposition \\
\hline & $\mathrm{SiH}_{4}: 20$ & $\mathrm{SiH}_{4}: 8$ \\
Gas flow (sccm) & $\mathrm{H}_{2}: 100$ & $\mathrm{~N}_{2} \mathrm{O}: 200$ \\
& $\mathrm{Ar}: 30$ & $\mathrm{Ar}: 30$ \\
rf power (W) & & $\mathrm{H}_{2}: 30$ \\
rf-bias power (W) & 900 & 900 \\
Process pressure (Torr) & 0.00005 & $200-400$ \\
Substrate temperature $\left({ }^{\circ} \mathrm{C}\right)$ & 350 & 0.00005 \\
Refractive index at 633 $\mathrm{nm}$ & 4.40 & 350 \\
\hline \hline
\end{tabular}

functional chamber for deposition dielectric materials and it can tune refractive index of materials by tuning the ratio of process gasses. This method can simplify the autocloning process to only one-step and apply this method to adjust the photonic band gap. Electron-beam exposure was carried out in the Leica Weprint-200 shaped-beam stepper. The electron beam energy was $40 \mathrm{keV}$ with a beam size of $20 \mathrm{~nm}$. Critical dimensions (CD) were evaluated with an in-line SEM (Hitachi S-6280) or cross-sectional SEM (Hitachi S-4000). And other process parameters are shown in Table I.

\section{RESULTS AND DISCUSSION}

Many factors affect the autocloning process in HDPCVD, such as ICP power, rf-bias power, chamber pressure, substrate temperature, and the etching-depth and period of underlying grooves. The step coverage is an important parameter in CVD processes and also a key factor to the autocloning method. Step coverage depends on arriving angle and surface mobility during CVD processes. The surface mobility can be improved by decreasing the chamber pressure that can increase the mean-free-path of source materials. Raising the substrate temperature can also increase surface mobility. In our experiment, we used a low process pressure (about $10^{-4}-10^{-5}$ Torr) and the substrate temperature was raised to $350{ }^{\circ} \mathrm{C}$. Sputter etching is another key factor to autocloning process. The arriving angle can be modified by the etching of material deposited on the underlying grooves. We raised the ICP power to $900 \mathrm{~W}$ in order to maintain a high-density plasma and provide sufficient argon ions for heavy ion-bombardment.

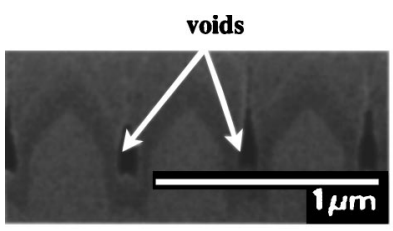

(a) (b)
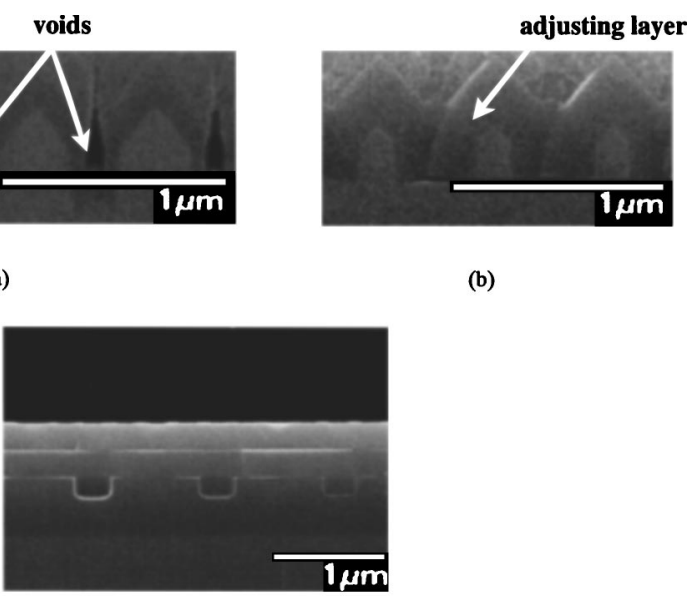

(c)

FIG. 2. (a) Initial deposition without $\mathrm{SiO}_{2}$ adjusting-layer; (b) Initial deposition with $\mathrm{SiO}_{2}$ adjusting-layer; (c) deposited by conventional PECVD with 300 mTorr chamber pressure.

The mechanism of the autocloning effect of HDP-CVD is shown in Fig. 1. CVD deposition and sputter etching are carried out alternately as described below: First, deposition of films by CVD processes as shown in Figs. 1(a)-1(c). Secondly, sputter etching and corner chopping with an angulardependent etching as shown in Figs. 1(d)-1(f). Sputter etching is a physical etching process without chemical reaction with thin-films and usually operates under low pressure with argon ion plasma. The sputter-etching rate of step corner will be faster than it is for removing thin films from the surface. Hence the slope angle of the step corner will be retained. By balancing these two phenomena, the surface shapes remain the same before and after a cycle of the processes. Therefore, the corrugated shapes are duplicated one after another.

Here we stacked autocloned structures composed of $a-\mathrm{Si} / \mathrm{SiO}_{2}$ multilayers using HDP-CVD systems under different conditions. We fabricated periodic grooves with $0.2-$ $0.5 \mu \mathrm{m}$ in period and $0.2-0.4 \mu \mathrm{m}$ in depth on Si wafers by using electron-beam lithography and reactive ion etching. Figures 2(a) and 2(b) show the difference of initial-layer pattern between samples without a $0.4 \mu \mathrm{m} \mathrm{SiO}_{2}$ adjusting-layer and those with an adjusting layer, respectively. Obviously, voids were formed in trenches without the adjusting-layer. As shown in Fig. 2(c), we attempted to use conventional

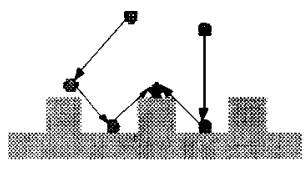

(a)

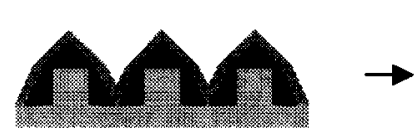

(d)

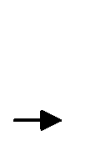

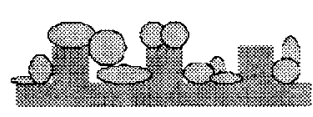

(b)

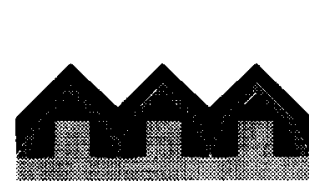

(e)

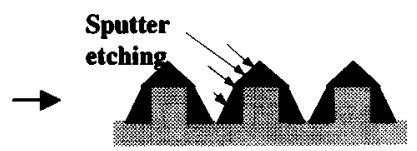

(c)

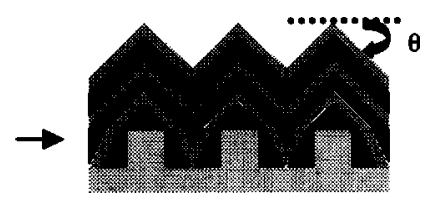

(f)
FIG. 1. Mechanism of autocloning method by using HDP-CVD (a) source gases or vapor precursors reach and migrate on the substrate surface; (b) solid by-products form nuclei and then nuclei grow into islands; (c) islands merge into the continuous thin film and then are etched by anisotropic sputter-etching; (d) grooves are filled from the bottom; (e) multilayer stacking of different materials; (f) autocloning photonic crystal is formed. 


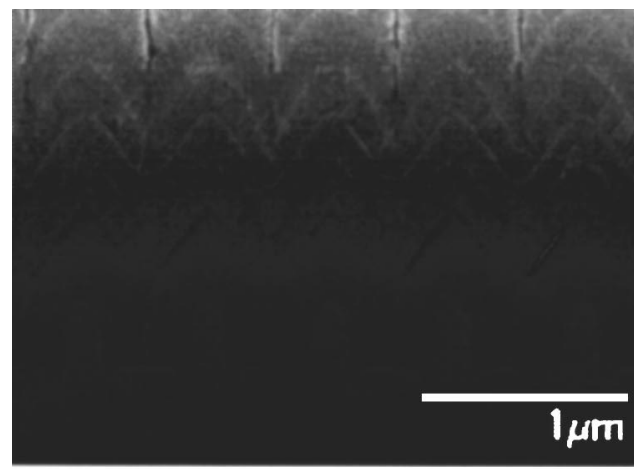

(a)

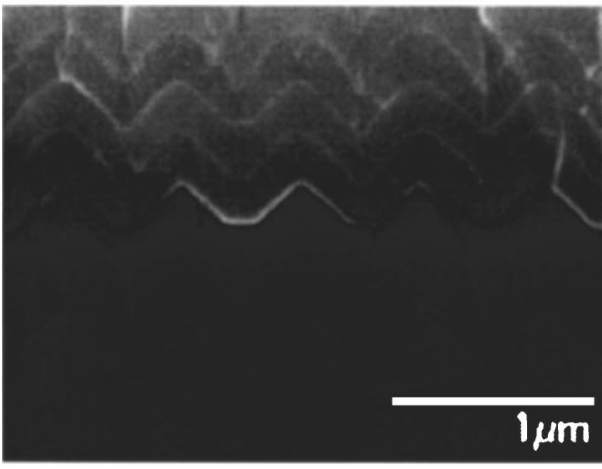

(b)

FIG. 3. Autocloning photonic crystals deposited by HDP-CVD with grooves of (a) $0.4 \mu \mathrm{m}$ and (b) $0.2 \mu \mathrm{m}$ in depth.

PECVD to deposit autocloned structure but were unsuccessful. We attribute to the large chamber pressure and less ion bombardment. Figures 3(a) and 3(b) show self-shaping processes for grooves with $0.2 \mu \mathrm{m}$ and $0.4 \mu \mathrm{m}$ in depth, respectively. Results show that the depth of initial grooves has little effect on the corrugation slope.

Using the same deposition conditions as shown in Table I, we changed the rf-bias power from $200 \mathrm{~W}$ to $400 \mathrm{~W}$. As shown in Fig. 4, the wavy shape and the slope angle is highly modulated by changing the rf-bias power. Figure 4(a) shows the top of the wavy shape becoming rounded at $200 \mathrm{~W}$ bias power. When the bias power is raised to $300 \mathrm{~W}$, the top of the wavy shape becomes sharp and the standard 2D autocloned photonic crystals can be formed as shown in Fig. 4(b). Since the slope of autocloned 2D structures fabricated by $300 \mathrm{~W}$ bias power is almost constant, the thickness of each layer is uniform. As a result, the average refractive index in the direction perpendicular to the film is highly modulated by tuning stacked thickness of alternating-layers, and generally the structures have large band gaps in the perpendicular direction. However, the average refractive index in the in-plane direction is almost constant, and the band gap is small or no band gap is observed in the in-plane direction.

To achieve full 3D band gaps by the drilled autocloned structure, a steep slope is required. Kawakami's group increased the freedom of the shaping process by introducing

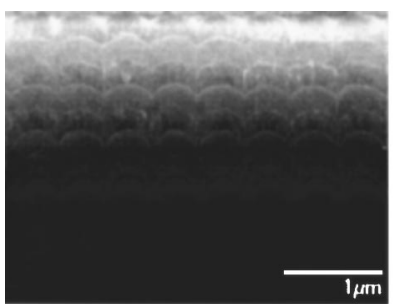

(a)

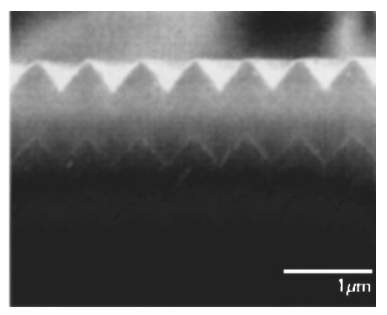

(b)

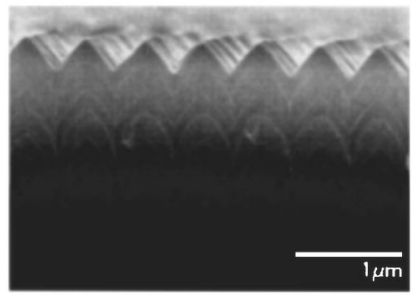

(c)

FIG. 4. Autocloning photonic crystals deposited by HDP-CVD with (a) bias power $=200 \mathrm{~W}$, (b) bias power $=300 \mathrm{~W}$, and (c) bias power $=400 \mathrm{~W}$.

reactive ion etching on $\mathrm{SiO}_{2}$ layer and fabricated a novel autocloned photonic crystals containing $\mathrm{SiO}_{2}$ arrowhead pillars in Si layer. These had a strong modulation of the effective refractive index in the lateral directions and exhibit larger bandgaps in those directions. ${ }^{12,14}$ But the complicated process contains three steps, $\mathrm{SiO}_{2}$ film deposition, $\mathrm{SiO}_{2}$ film etching by RIE and then $a$-Si film deposition. In this article, we achieve a similar result by only raising bias power to 400 $\mathrm{W}$ as shown in Fig. 4(c). The wavy shape of $\mathrm{SiO}_{2}$-layer is highly modulated by bias power and the maximum angle of slope is about $70^{\circ}$, which is steeper than that of general autocloned structures. In the process, the oblique degree and wavy shape in Si-layer almost are almost unchanged. The reason may be caused by different etching properties of materials. We successfully fabricate a novel autocloned photonic crystals structure, which contains $\mathrm{SiO}_{2}$ arrowhead pillars in the Si film by utilizing the more powerful sputter-etching effect without introducing additional processes. The definition of corrugation angle $\theta$ is shown in Fig. 1(f). As shown in Fig. 4(a), the corrugation angle was about $25^{\circ}$ when the bias power was $200 \mathrm{~W}$. As the bias power increased to $300 \mathrm{~W}$, Fig. 4(b) shows the corrugation angle increased to about $45^{\circ}$. Figure $4(c)$ shows the corrugation angle increased to about $70^{\circ}$ with $\mathrm{SiO}_{2}$ arrowhead pillars in the Si layer as the bias power increased to $400 \mathrm{~W}$.

Furthermore, the HDP-CVD method has better stepcoverage than the sputtering method and thus can be used to fabricate autocloning structures with small feature-sizes. Figure 5 shows autocloning structures deposited on the corrugated structures with period from $200 \mathrm{~nm}$ to $500 \mathrm{~nm}$. Results indicate autocloned structures can be retained for different underlying structure periods. This method enables autocloned structures for periods of $200 \mathrm{~nm}$. From the abovementioned, we have developed a simpler and more reliable autocloning method by using the HDP-CVD method. 


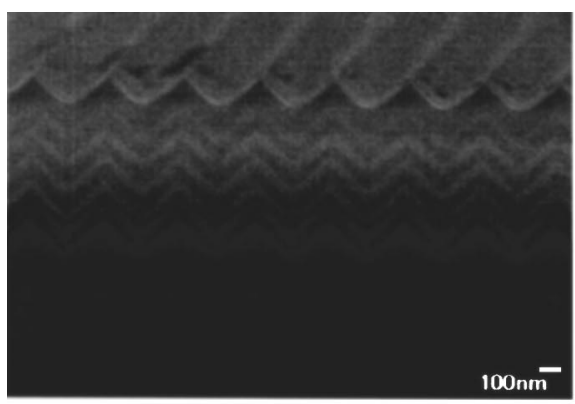

(a)

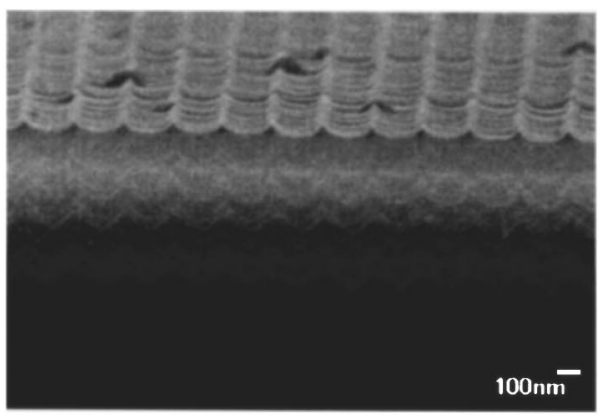

(c)

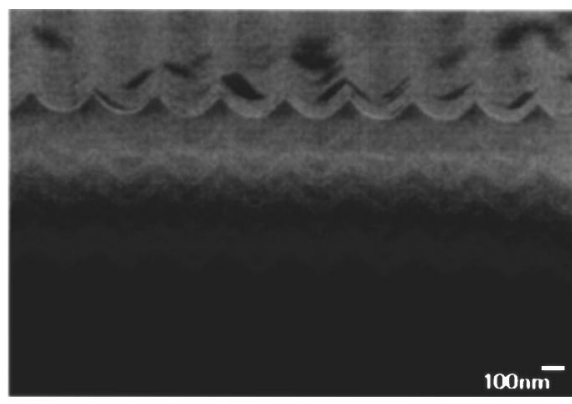

(b)

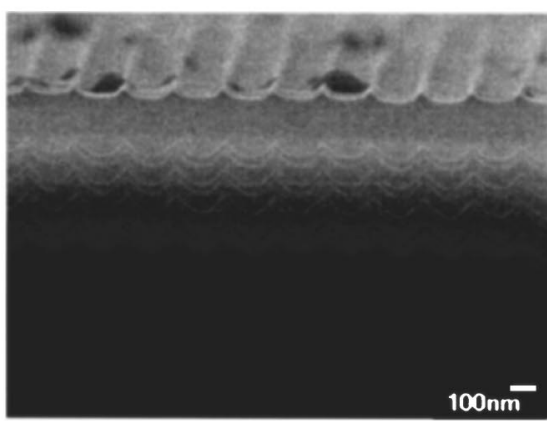

FIG. 5. Autocloning photonic crystals deposited by HDP-CVD on the corrugated structures with period of (a) 500 $\mathrm{nm}$, (b) $400 \mathrm{~nm}$, (c) $300 \mathrm{~nm}$, and (d) $200 \mathrm{~nm}$.

\section{CONCLUSION}

We successfully proposed a new method for autocloned photonic crystals fabrication and also demonstrated the selfshaping mechanism and conditions. The HDP-CVD method was used as an alternative to the rf-bias sputtering method for fabrication of autocloned photonic crystals. We successfully preserved the periodic surface corrugation after deposition of multilayer stacks under appropriate chemical vapor deposition conditions. The freedom of the shaping process was enhanced by simply rasing bias power of the autocloning process, and thus created the autocloned structures having strong modulation of the effective refractive index in the lateral direction. We successfully fabricated a novel autocloned photonic crystals structure, which contains $\mathrm{SiO}_{2}$ arrowhead pillars in the Si film without introducing additional processes. Furthermore, the HDP-CVD method has better step-coverage than the sputtering method that can be used to fabricate autocloning structures with period down to $200 \mathrm{~nm}$. The method allows photonic bands of autocloned photonic crystals to be designed with greater controllability and simpler fabrication processes. (d)
${ }^{1}$ E. Yablonovitch, Phys. Rev. Lett. 58, 2059 (1987).

${ }^{2}$ S. Kawakami, Electron. Lett. 33, 1260 (1997).

${ }^{3}$ T. Kawashima, T. Sato, K. Miura, Y. Ohtera, N. Ishino, and S. Awakami, in Proceedings of the International School Quantum Electronics, 2000, pp. 115-122.

${ }^{4}$ Y. Ohtera, T. Sato, T. Kawashima, T. Tamamura, and S. Kawakami, Electron. Lett. 35, 1271 (1999).

${ }^{5}$ H. Kosaka, T. Kawashima, A. Tomita, M. Notomi, T. Tamamura, T. Sato, and S. Kawakami, Phys. Rev. B 58, R10096 (1998).

${ }^{6}$ H. Kosaka, T. Kawashima, A. Tomita, M. Notomi, T. Tamamura, T. Sato, and S. Kawakami, J. Lightwave Technol. 17, 2032 (1999).

${ }^{7}$ H. Kosaka, T. Kawashima, A. Tomita, M. Notomi, T. Tamamura, T. Sato, and S. Kawakami, Appl. Phys. Lett. 74, 1212 (1999).

${ }^{8}$ O. Hanaizumi, Y. Ohtera, T. Sato, and S. Kawakami, Appl. Phys. Lett. 74, 777 (1999)

${ }^{9}$ T. Sato, Y. Ohtera, N. Ishino, K. Miura, and S. Kawakami, IEEE J. Quantum Electron. 38, 904 (2002).

${ }^{10}$ M. Notomi, T. Tamamura, T. Kawashima, and S. Kawakami, Appl. Phys. Lett. 77, 4256 (2000).

${ }^{11}$ E. Kuramochi, M. Notomi, I. Yokohama, J. Takahashi, C. Takahashi, T. Kawashima, and S. Kawakami, J. Appl. Phys. 93, 8848 (2003).

${ }^{12}$ S. Kawakami, T. Kawashima, and T. Sato, Appl. Phys. Lett. 74, 463 (1999).

${ }^{13}$ Hong Xiao, Introduction to Semiconductor Manufacturing Technology (Prentice-Hall, Englewood Cliffs, 2001).

${ }^{14}$ T. Kawashima, T. Sato, Y. Ohtera, and S. Kawakami, IEEE J. Quantum Electron. 38, 899 (2002). 